\title{
Non-linear structure formation in cosmologies with early dark energy
}

\author{
M. Bartelmann ${ }^{1}$, M. Doran ${ }^{2}$, and C. Wetterich ${ }^{2}$ \\ ${ }^{1}$ Zentrum für Astronomie, ITA, Universität Heidelberg, Albert-Überle-Str. 2, 69120 Heidelberg, Germany \\ e-mail: mbartelmann@ita.uni-heidelberg.de \\ 2 Institut für Theoretische Physik, Universität Heidelberg, Philosophenweg 16, 69120 Heidelberg, Germany \\ Received 26 July 2005 / Accepted 28 February 2006
}

ABSTRACT

\begin{abstract}
We argue that a few per cent of "Early Dark Energy" can be detected by the statistics of nonlinear structures. The presence of Dark Energy during linear structure formation is natural in models where the current low Dark-Energy density is related to the age of the Universe rather than a new fundamental small parameter. Generalisation of the spherical collapse model shows that the linear collapse parameter $\delta_{\mathrm{c}}$ is lowered. The corresponding relative enhancement of weak gravitational lensing on arc-minute scales lowers the value of $\sigma_{8}$ inferred from a given lensing amplitude, as compared to $\Lambda \mathrm{CDM}$. In the presence of Early Dark Energy, structures grow slower, such that for a given $\sigma_{8}$, the number of galaxies and galaxy clusters is substantially increased at moderate and high redshift. For realistic models, the number of clusters detectable through their thermal Sunyaev-Zel'dovich effect at redshift unity and above, e.g. with the Planck satellite, can be an order of magnitude larger than for $\Lambda \mathrm{CDM}$.
\end{abstract}

Key words. cosmology: theory - cosmology: early Universe - cosmology: large-scale structure of Universe

\section{Introduction}

According to observations of Supernovae Ia (SNe Ia) (Riess et al. 2004), the Cosmic Microwave Background (CMB) (Spergel et al. 2003; Readhead et al. 2004; Goldstein et al. 2003; Rebolo et al. 2004), and Large Scale Structure (LSS) (Tegmark et al. 2004; Hawkins et al. 2003), the expansion of our Universe is accelerating today. A plausible candidate to drive such an acceleration is Dark Energy (DE) which may be described by an effective (Doran \& Jäckel 2002) scalar field (Wetterich 1988; Ratra \& Peebles 1988; Caldwell et al. 1998; Caldwell 2002). Such scalar field models have been shown to admit attractor solutions (Wetterich 1988; Ratra \& Peebles 1988; Liddle \& Scherrer 1999; Zlatev \& Steinhardt 1999) where the DE component "tracks" the dominant component of the cosmological fluid. It is therefore natural to assume that DE might be non-negligible during extended periods of the evolution of our Universe.

If the DE fraction is varying mildly during the entire history of the Universe, the present tiny DE density can be related to the huge age of the Universe. Such an "Early DE" contribution has been shown to influence the CMB (Doran et al. 2001a; Caldwell et al. 2003) and linear structure growth (Ferreira \& Joyce 1998; Doran et al. 2001b). From the CMB and LSS data, one infers a current limit on the DE contribution $\Omega_{d}$ during the recombination and structure formation of $\Omega_{\mathrm{d}} \lesssim 10 \%$. Intriguingly, the effect of Early DE on structure formation is particularly strong because it influences the entire structure formation period in contrast to a cosmological constant model or any other model of DE with $\Omega_{\mathrm{d}} \rightarrow 0$ at earlier times.

The non-linear structure growth in DE scenarios has been the subject of several investigations (see e.g. Wang \& Steinhardt 1998; Ma et al. 1999; Linder \& Jenkins 2003). In contrast to these works, we will focus on the implications of an Early DE component. This has two major effects. First, Early DE lowers the linear growth rate for structures. As a consequence, for a given present amplitude $\sigma_{8}$, the amplitude of structures at high redshift is higher, when compared to $\Lambda \mathrm{CDM}$. Second, on the non-linear level, there is more structure than one would expect from the linear amplitude in $\Lambda \mathrm{CDM}$. In consequence, the abundance of collapsed objects is considerably higher at redshifts $z \sim 1$ than in a standard $\Lambda C D M$ universe. The decisive quantity for the number of non-linear objects is the probability for a region containing massive particles with a total mass $M$ to collapse at a redshift $z_{\mathrm{c}}$. It is proportional to

$\exp \left[-\frac{\delta_{\mathrm{i}}^{2}}{2 \sigma_{\mathrm{i}, R}^{2}}\right]$,

where the density contrast at early times (e.g. at matter-radiation equality), $\delta_{\mathrm{i}}$, is chosen such that the region collapses by redshift $z_{\mathrm{c}}$, and the variance at early times, $\sigma_{\mathrm{i}, R}$, is evaluated on a linear scale corresponding to the mass, $R \propto(M / \rho)^{1 / 3}$. For a given current fluctuation amplitude $\sigma_{8}$, the value of $\sigma_{\mathrm{i}, R}$ is larger in cosmologies with Early DE than in $\Lambda$ CDM. Since $\delta_{\mathrm{i}}$ only mildly depends on the detailed cosmology, this enhancement of $\sigma_{\mathrm{i}}$ explains the enhancement of non-linear structures relative to $\Lambda \mathrm{CDM}$. Conversely, the value of $\sigma_{8}$ required to explain the number counts of non-linear objects or weak gravitational lensing on small angular scales is smaller for models with Early DE than for $\Lambda$ CDM. Comparison of the linear fluctuation amplitude (e.g. from galaxy correlations or weak gravitational lensing on large angular scales) with the power in non-linear structures could thus provide clear evidence for the presence of Early DE.

The crucial quantity for Early DE is its average density fraction during structure formation, $\Omega_{\mathrm{d}, \mathrm{sf}}$ (see Doran et al. $2001 \mathrm{~b}$ for a precise definition). As an example of the high sensitivity of non-linear structures to $\Omega_{\mathrm{d}, \mathrm{sf}}$, we present two models with $\Omega_{\mathrm{d}, \mathrm{sf}}=0.04$. We should also mention that a possible coupling between DE and Dark Matter (DM) could modify our results, but will be neglected in this work. 
We use a particularly simple and direct parametrisation of the Dark Energy evolution (Wetterich 2004). The parameters are the amount of Dark Energy today, $\Omega_{\mathrm{d}, 0}$; the equation-of-state parameter today, $w_{0}$; and the amount of DE at early times, $\Omega_{\mathrm{d}, \mathrm{e}}$, to which it asymptotes for $z \rightarrow \infty$. One important feature of our parametrisation is a non-vanishing DE contribution during recombination and structure formation. As an illustration, we pick two models at random from a Monte-Carlo chain. We select models for which $\sigma_{8}$ is close to 0.8 , the optical depth is $\tau<0.2$, and the spectral indices of initial scalar perturbations are $n=0.99$ and $n=1.05$, respectively. The data we use for the Monte-Carlo chains are CMB (Spergel et al. 2003; Readhead et al. 2004; Goldstein et al. 2003; Rebolo et al. 2004), LSS (Tegmark et al. 2004), and SNe Ia (Riess et al. 2004) data, thus both models describe current observations well. The parameters for model (I) are: $\Omega_{\mathrm{m}, 0} h^{2}=0.146, \Omega_{\mathrm{b}} h^{2}=0.026, h=0.67$, $n=1.05, \tau=0.18, w_{0}=-0.93$, and $\Omega_{\mathrm{d}, \mathrm{e}}=2 \times 10^{-4}$, leading to an effective Dark Energy contribution during structure formation (Doran et al. 2001b) of $\Omega_{\mathrm{d}, \mathrm{sf}}=0.04$ and $\sigma_{8}=0.82$. Model (II) is given by $\Omega_{\mathrm{m}, 0} h^{2}=0.140, \Omega_{\mathrm{b}, 0} h^{2}=0.023, h=0.62$, $n=0.99, \tau=0.18, w_{0}=-0.99$ and $\Omega_{\mathrm{d}, \mathrm{e}}=8 \times 10^{-4}$, leading to $\Omega_{\mathrm{d}, \mathrm{sf}}=0.04$ and $\sigma_{8}=0.78$. We compare these models to $\Lambda \mathrm{CDM}$ with $\Omega_{\mathrm{m}, 0}=0.3, \Omega_{\Lambda, 0}=0.7, h=0.65$, and $\sigma_{8}=0.84$.

\section{Spherical collapse with Early DE}

We repeat in this section the calculation of the well-known spherical-collapse parameter $\delta_{\mathrm{c}}$ for cosmological models with Early DE. The main difference in comparison to the numerous earlier calculations (e.g. Lahav et al. 1991; Kitayama \& Suto 1996; Wang \& Steinhardt 1998; Weinberg \& Kamionkowski 2003; Horellou \& Berge 2005; Maor \& Lahav 2005; Mota \& van de Bruck 2004) is that the presence of non-negligible DE at early times does not allow the common simplification that only the matter density is important at sufficiently early times of structure formation. Rather, we must take into consideration the idea that a form of energy, which has a variable equation of state, contributes early on to structure formation. The course of the calculation is thus largely the same as in other studies of the subject, but the terms that need to be kept are different. The main result of this section is that the coefficients $A$ and $B$ appearing in the first-order Taylor expansion (32) of the overdensity $\Delta$ inside collapsing spherical perturbations acquire some dependence on the DE's equation-of-state. A second important effect is that the Early DE increases the expansion rate at early times, causing a decrease in the linear growth factor $D_{+}$. Both effects will now be quantified.

\subsection{Solutions for early times}

The qualitative features of the effects of Early DE can be understood analytically within the spherical collapse model. We consider a homogeneous, spherical overdensity that expands, reaches its maximum radius ("turn-around") at a scale factor $a_{\mathrm{ta}}$, and then collapses to reach virial equilibrium. We restrict the consideration to the matter-dominated era, i.e. we neglect the very slow growth of a perturbation within the horizon during the radiation-dominated era.

Following Wang \& Steinhardt (1998), we refer all quantities to their values at turn-around. Let $\omega$ and $\lambda$ be the density parameters, at turn-around, of the (Dark) Matter and the DE, respectively. The cosmological scale factor $x$ and the physical radius $y$ of the density perturbation are normalised to unity at turn-around,

$x \equiv \frac{a}{a_{\mathrm{ta}}}, \quad y \equiv \frac{R}{R_{\mathrm{ta}}}$.
The spherical collapse of the perturbation is then described by the two Friedmann equations

$\dot{x}=\left[\frac{\omega}{x}+\lambda x^{2} g(x)+(1-\omega-\lambda)\right]^{1 / 2}$,
$\ddot{y}=-\frac{\omega \zeta}{2 y^{2}}-\frac{1+3 \omega(x)}{2} \lambda g(x) y$,

where dots denote derivatives with respect to the dimensionless time parameter

$\tau \equiv H_{\mathrm{ta}} t$.

The parameter $\zeta$ quantifies the matter overdensity at turn-around in a spherical volume, which will collapse at a later, pre-defined time. It is determined by solving Eq. (4) with the boundary conditions $y=0$ at $\tau=0$ and $\dot{y}=0$ at turn-around and by requiring that $y=1$ at turn-around. In other words, once the collapse time (or redshift) is fixed to the time when the perturbation formally collapses to zero radius, $\zeta$ is chosen such that the maximum radius is reached at the turn-around time, given the boundary conditions. The function $g(x)$ quantifies the change in the DE density with $x$ relative to turn-around,

$g(x)=\exp \left[-3 \int_{0}^{\ln x}\left[1+w\left(x^{\prime}\right)\right] \mathrm{d} \ln x^{\prime}\right] \equiv x^{-3(1+\bar{w})}$,

where $\bar{w}$ is a suitably averaged equation-of-state parameter. For constant $w$, one has $\bar{w}=w$, but in general $w$ and $\bar{w}$ are functions of $\tau$ or, equivalently, $x$.

We assume that the DE density is always sufficiently smaller than the matter density at early times, i.e.

$g(x) \ll x^{-3}, \quad x^{3} g(x) \ll 1 \quad$ for $\quad x \rightarrow 0$.

Then, from Eq. (3),

$\mathrm{d} \tau \approx \sqrt{\frac{x}{\omega}}\left(1-\frac{1-\omega-\lambda}{2 \omega} x\right) \mathrm{d} x$

for $x \ll 1$, and thus, at early times,

$\sqrt{\omega} \tau \approx \frac{2}{3} x^{3 / 2}\left[1-\frac{3}{10} \frac{1-\omega-\lambda}{\omega} x\right]$

showing no effect of DE to second order in $x$.

Upon multiplication with $2 \dot{y}$, Eq. (4) becomes

$\frac{\mathrm{d}\left(\dot{y}^{2}\right)}{\mathrm{d} \tau}=\omega \zeta \frac{\mathrm{d}(1 / y)}{\mathrm{d} \tau}-\frac{1+3 w}{2} \lambda g(x) \frac{\mathrm{d}\left(y^{2}\right)}{\mathrm{d} \tau}$.

Integration gives

$\dot{y}^{2}=\frac{\omega \zeta}{y}-\lambda \int_{0}^{y}\left[1+3 w\left(x^{\prime}\right)\right] g\left(x^{\prime}\right) y^{\prime} \mathrm{d} y^{\prime}+C$,

with the integration constant $C$ set by the requirement at turnaround that

$\left.\dot{y}\right|_{y=1}=0$,

which is satisfied if

$C=\lambda \int_{0}^{1}\left[1+3 w\left(x^{\prime}\right)\right] g\left(x^{\prime}\right) y^{\prime} \mathrm{d} y^{\prime}-\omega \zeta$.

When inserted back into Eq. (11), this yields

$\dot{y}^{2}=\omega \zeta\left(\frac{1}{y}-1\right)+\lambda \int_{y}^{1}\left[1+3 w\left(x^{\prime}\right)\right] g\left(x^{\prime}\right) y^{\prime} \mathrm{d} y^{\prime}$. 
We will have to study Eq. (14) at very early times, i.e. for $x \rightarrow 0$ and $y \rightarrow 0$. Within those limits, the integral in Eq. (14) will either converge or diverge as $x$ and $y$ approach zero. Suppose the integral converges,

$\lim _{y \rightarrow 0} \int_{y}^{1}(1+3 w) g(x) y \mathrm{~d} y \equiv I<\infty$.

Then, for $y \rightarrow 0$, Eq. (14) can be approximated by

$\frac{\sqrt{y} \mathrm{~d} y}{\sqrt{1-A y}}=\sqrt{\omega \zeta} \mathrm{d} \tau, \quad$ where $\quad A \equiv 1-\frac{\lambda I}{\omega \zeta}$,

which can be integrated after expanding the denominator into a Taylor series,

$\sqrt{\omega \zeta \tau} \approx \int_{0}^{y} \sqrt{y}\left(1+\frac{A y}{2}\right)=\frac{2}{3} y^{3 / 2}\left(1+\frac{3 A}{10} y\right)$.

For example, if the DE is described by a cosmological constant, $w=-1$ and $g(x)=1$, thus $I=-1$ and $A=1+\lambda / \omega \zeta$.

The integral $I$ defined in Eq. (15) will diverge if $g(x)$ increases steeply as $x \rightarrow 0$. Then, it will be dominated by the behaviour of its integrand for $x \rightarrow 0$. Let

$\lim _{x \rightarrow 0} w(x) \equiv w_{\text {ini }}, \quad$ and $\quad \lim _{x \rightarrow 0} g(x) \equiv \gamma x^{-3\left(1+w_{\text {ini }}\right)}$,

and let us further assume $y$ to be proportional to $x$ at early times, $y=\alpha x$, which will be justified in hindsight. The constant $\gamma \ll 1$ because the DE density was assumed in Eq. (7) to be less than the matter density at early times. Then,

$$
\begin{aligned}
I & \approx\left(1+3 w_{\text {ini }}\right) \alpha^{2} \gamma \lim _{x \rightarrow 0} \int_{x}^{1} x^{\prime-3\left(1+w_{\text {ini }}\right)} x^{\prime} \mathrm{d} x^{\prime} \\
& \approx \alpha^{2} \gamma \lim _{x \rightarrow 0}\left(\left.x^{\prime-1-3 w_{\text {ini }}}\right|_{x} ^{1}\right) \\
& \approx \frac{\gamma \alpha^{3\left(1+w_{\text {ini }}\right)}}{y^{1+3 w_{\text {ini }}}}
\end{aligned}
$$

$I$ is thus expected to diverge if $w_{\text {ini }} \geq-1 / 3$. With Eq. (14), this yields, for early times,

$\dot{y}=\left[\frac{\omega \zeta}{y}-\omega \zeta+\lambda \gamma \frac{\alpha^{3\left(1+w_{\text {ini }}\right)}}{y^{1+3 w_{\text {ini }}}}\right]^{1 / 2}$.

According to Eq. (7), $w_{\text {ini }} \leq 0$, because otherwise the DE density would grow above the matter density. Let $w_{\text {ini }}=0$ first, then Eq. (20) implies

$$
\begin{aligned}
\dot{y} & =\left[\frac{\omega \zeta+\lambda \gamma \alpha^{3}}{y}-\omega \zeta\right]^{1 / 2} \\
& \approx \sqrt{\frac{\omega \zeta+\lambda \gamma \alpha^{3}}{y}}\left(1-\frac{\omega \zeta}{2\left(\omega \zeta+\lambda \gamma \alpha^{3}\right)} y\right),
\end{aligned}
$$

which can be integrated to give

$\sqrt{\omega \zeta} \tau \approx \frac{2}{3} y^{3 / 2} \sqrt{B}\left(1+\frac{3}{10} B y\right), \quad B \equiv \frac{\omega \zeta}{\omega \zeta+\lambda \gamma \alpha^{3}}$.

If $-1 / 3<w_{\text {ini }}<0$, we can replace $B$ by

$B=\frac{\omega \zeta}{\omega \zeta+\lambda \gamma \alpha^{3\left(1+w_{\text {ini }}\right)} y^{-3 w_{\text {ini }}}} \approx 1-\frac{\lambda \gamma}{\omega \zeta} \alpha^{3\left(1+w_{\text {ini }}\right)} y^{-3 w_{\text {ini }}}$

to account for the gentle change in the coefficient $B$ with $y$.
Equating $\sqrt{\zeta}$ times Eq. (9) to Eq. (17) and squaring yields

$\zeta x^{3}\left(1-\frac{3}{5} \frac{1-\omega-\lambda}{\omega} x\right) \approx y^{3}\left(1+\frac{3 A}{5} y\right)$

while the same procedure applied to Eq. (22) implies

$\zeta x^{3}\left(1-\frac{3}{5} \frac{1-\omega-\lambda}{\omega} x\right) \approx y^{3} B\left(1+\frac{3 B}{5} y\right)$.

Now, $B \approx 1$ to the lowest order because $\gamma \ll 1$ and $y \ll 1$ at early times, thus

$y \approx \zeta^{1 / 3} x, \quad \alpha=\zeta^{1 / 3}$

to the lowest order, and the earlier assumption $y \approx \alpha x$ is verified. The definition of $B$ in Eqs. (22) and (23) then simplifies to

$B \approx 1-\frac{\lambda \gamma \zeta^{w_{\text {ini }}} y^{-3 w_{\text {ini }}}}{\omega}$

To illustrate the procedure described above, let us assume $w(a)=$ $-a$, or $w(x)=-a_{\mathrm{ta}} x$, for which $g(x)$ from Eq. (6) becomes

$g(x)=\exp \left[-3 \int_{0}^{\ln x}\left(1-a_{\mathrm{ta}} x^{\prime}\right) \mathrm{d} \ln x^{\prime}\right]=\frac{\mathrm{e}^{3 a_{\mathrm{ta}}(x-1)}}{x^{3}}$.

This yields

$$
\begin{aligned}
\int_{y}^{1}\left[1+3 w\left(x^{\prime}\right)\right] g\left(x^{\prime}\right) y^{\prime} \mathrm{d} y^{\prime} & \approx \alpha^{2}\left[\frac{\mathrm{e}^{3 a_{\mathrm{ta}}(x-1)}}{x}-1\right] \\
& \approx \frac{\alpha^{2} \mathrm{e}^{-3 a_{\mathrm{ta}}}}{x} \approx \frac{\alpha^{3} \mathrm{e}^{-3 a_{\mathrm{ta}}}}{y} .
\end{aligned}
$$

Following Eq. (18), we have

$w_{\text {ini }}=0, \quad \gamma=\mathrm{e}^{-3 a_{\mathrm{ta}}}$,

confirming that $\gamma \ll 1$ for typical values of $a_{\mathrm{ta}} \lesssim 1$. Thus, Eq. (29) agrees with the more general result Eq. (19).

\subsection{Overdensity and linear density contrast}

Since the background matter density changes in proportion to $x^{-3}$, while the matter density inside the perturbation is proportional to $y^{-3}$, the overdensity inside the perturbation obeys

$\Delta=\frac{\zeta x^{3}}{y^{3}}$

which, according to Eq. (24) and Eq. (25), is

$\Delta \approx\left\{\begin{array}{l}1+\frac{3 A}{5} y+\frac{3}{5} \frac{1-\omega-\lambda}{\omega} x \text { for } \omega_{\text {ini }}<-1 / 3 \\ 1+\frac{3 B}{5} y+\frac{3}{5} \frac{1-\omega-\lambda}{\omega} x \text { else }\end{array}\right.$

at early times. The two solutions join at $w_{\text {ini }}=-1 / 3$, as they should. For $w_{\text {ini }}=-1 / 3, I=0$ because $\left(1+3 w_{\text {ini }}\right)=0$ in Eq. (19), thus $B=1$. Also, $A \rightarrow 1$ for $w \rightarrow-1 / 3$ according to Eqs. (15) and (16).

The overdensity $\Delta_{\mathrm{V}}$ within virialised objects should only be very weakly affected by the (Early) DE because DM dominates the virialisation process. We verify this as described in Appendix A.

The linear density contrast $\delta$ can be used to relate the nonlinear overdensity $\Delta$ with the density that would result from the 


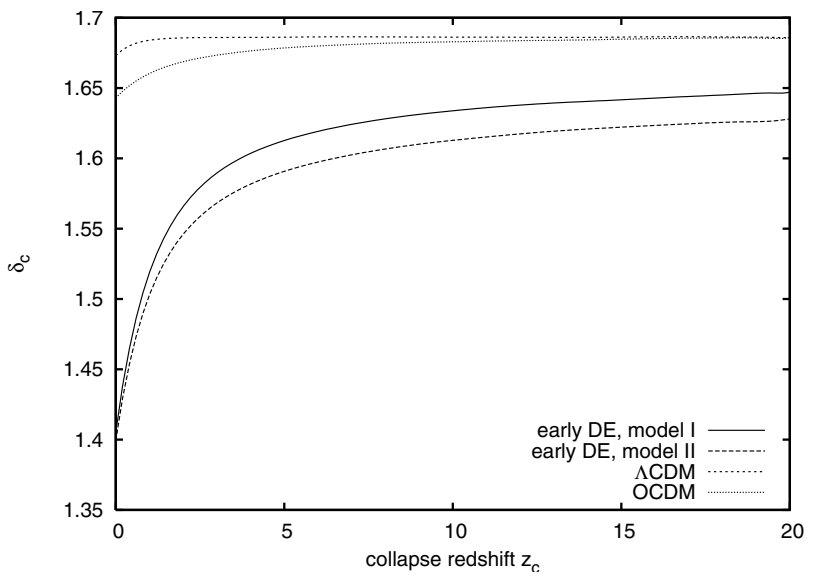

Fig. 1. The linear density contrast at collapse $\delta_{\mathrm{c}}$ is plotted as a function of the collapse redshift $z_{\mathrm{c}}$ for two models with Early DE and for $\Lambda C D M$ and OCDM for comparison. While the $\Lambda$ CDM and the OCDM models show very similar behaviour, with $\delta_{\mathrm{c}}$ near the "canonical" value of 1.686 , the Early DE models fall substantially below, in particular for low collapse redshifts.

linear evolution of the same initial perturbation within linear evolution. With the growth factor, $D_{+}(x)$ at a given time, or normalised scale factor, $x$, one has, at the collapse time $x_{\mathrm{c}}>1$,

$\delta_{\mathrm{c}}=\lim _{x \rightarrow 0}\left[\frac{D_{+}\left(x_{\mathrm{c}}\right)}{D_{+}(x)}[\Delta(x)-1]\right]$.

The linear density contrast at collapse time $\delta_{\mathrm{c}}$ is a crucial ingredient for the Press-Schechter and related mass functions (Press \& Schechter 1974; Sheth \& Tormen 1999). We shall explain below why $\delta_{\mathrm{c}}$ is substantially smaller for Early DE models as compared to $\Lambda \mathrm{CDM}$. This finding is a central component of our results.

\subsection{Results for $\delta_{\mathrm{c}}$}

We show the linear critical overdensity $\delta_{\mathrm{c}}$ as a function of $z_{\mathrm{c}}$ in Fig. 1. While the results range around $\delta_{\mathrm{c}}=3 / 5(3 \pi / 2)^{2 / 3} \approx$ 1.686 for $\Lambda$ CDM and OCDM, quite independent of the collapse redshift, they fall below for the Early DE models, dropping even to $\delta_{\mathrm{c}} \approx 1.4$ for $z_{\mathrm{c}}=0$.

We return to Eq. (33) to understand why $\delta_{\mathrm{c}}$ is lower for Early DE than for, e.g., the $\Lambda$ CDM model. It turns out numerically that the overdensity $(\Delta-1)$ is not changed much in the Early DE compared to the $\Lambda \mathrm{CDM}$ model. For a collapse redshift of $z_{\mathrm{c}}=0$, for instance, $(\Delta-1) / x$ is lower by only $4 \%$ in the Early DE than in the $\Lambda \mathrm{CDM}$ model. The main difference, however, is caused by the growth-factor ratio in Eq. (33), as shown in Fig. 2.

The curves show the growth factor divided by the scale factor, $D_{+}(a) / a$, as a function of $a$, for one of the Early DE models and for $\Lambda$ CDM. Both lower curves are normalised so that they start from unity at early times, as it should be according to Eq. (33). Obviously, the linear growth in Early DE falls behind that in $\Lambda \mathrm{CDM}$, beginning at an overdensity of the same amplitude. The reason is that the expansion rate in the Early DE models is higher at early times than in $\Lambda \mathrm{CDM}$, which increases the friction term $(\dot{a} / a)$ in the equation

$\ddot{\delta}+2 \frac{\dot{a}}{a} \dot{\delta}-4 \pi G \rho \delta=0$

governing the growth factor. As we shall see below, the reduced linear overdensity $\delta_{\mathrm{c}}$ expected in Early DE models has

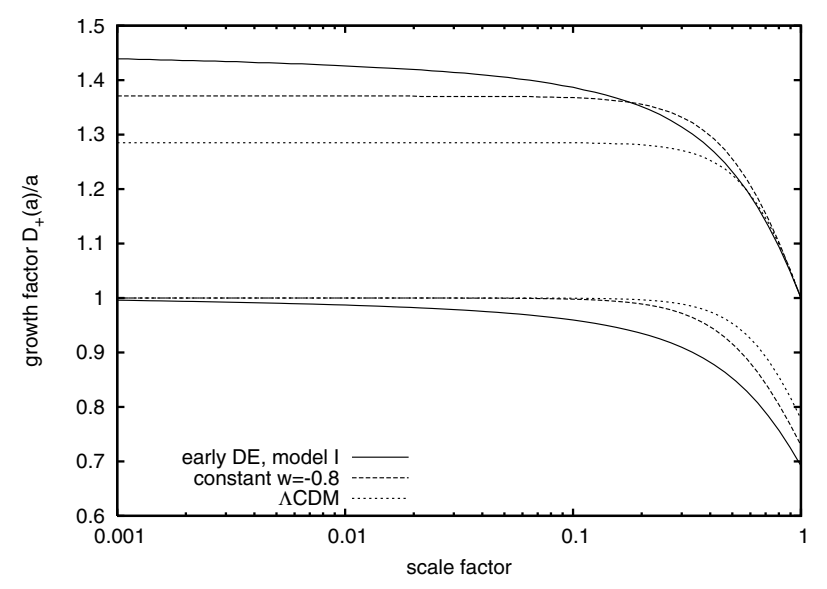

Fig. 2. The linear growth factor divided by the scale factor, $D_{+}(a) / a$, is shown here as a function of $a$ for an Early DE model, a model with constant $w=-0.8$, and for the $\Lambda \mathrm{CDM}$ model. The lower curves are normalised to unity at early times, i.e. they illustrate the growth of perturbations starting from the same density contrast in the two cosmologies. The upper curves are normalised to unity at present $(a=1)$, illustrating the growth of structures reaching the same level today in both cosmologies. Starting from the same amplitude, the growth in the Early DE model falls behind that in $\Lambda \mathrm{CDM}$, largely causing the reduction in the overdensity parameter $\delta_{\mathrm{c}}$. Structures have to grow earlier in Early DE models than in $\Lambda \mathrm{CDM}$ to reach the same fluctuation amplitude today.

pronounced consequences for nonlinear structure formation. We have verified that our numerical solution for $\delta_{\mathrm{c}}$ agrees with the values found for $\Lambda \mathrm{CDM}$ and for DE models with constant $w$ by, e.g., Lahav et al. (1991), Kitayama \& Suto (1996), Wang \& Steinhardt (1998) and Weinberg \& Kamionkowski (2003).

The two upper curves in Fig. 2 show the growth factor normalised to unity at present, i.e. reflecting the evolution back in time of structures reaching the same amplitude today. They show that structures need to grow earlier in Early DE models than in $\Lambda C D M$ models to reach the same level at the present time.

An analysis of Eq. (34) at early times reveals how the growth factor changes compared to $\Lambda$ CDM. It turns out that, for a flat model universe with Early DE, the growth factor $D_{+}(a)$ increases with the scale factor $a$ as

$D_{+}(a) \propto a^{n} \quad$ with $\quad n=1-\frac{3 \Omega_{\mathrm{d}}(a)}{5}$

for $a \ll 1$ (cf. Ferreira \& Joyce 1998). Considering a perturbation that already collapses at high redshift, the growth factor in $\Lambda \mathrm{CDM}$ can be approximated by $D_{+}(a) \propto a$. If the perturbation started from the same overdensity at matter-radiation equality $a_{\text {eq }}$ in a $\Lambda \mathrm{CDM}$ and an Early DE model, the linear density contrast at collapse $\delta_{\mathrm{c}}$ would fall behind in the Early DE, compared to the $\Lambda$ CDM model, by a factor of

$\left(\frac{a_{\mathrm{c}}}{a_{\mathrm{eq}}}\right)^{-3 \Omega_{\mathrm{d}, \mathrm{f}} / 5}$,

and thus

$\delta_{\mathrm{c}} \rightarrow 1.686\left(z_{\mathrm{c}} a_{\mathrm{eq}}\right)^{3 \Omega_{\mathrm{d}}\left(a_{\mathrm{eq}}\right) / 5}$

for high-redshift collapse. For example, at $a_{\mathrm{eq}} \approx 2 \times 10^{-4}$, $\Omega_{\mathrm{d}}\left(a_{\mathrm{eq}}\right) \approx 0.01$, and the linear density contrast $\delta_{\mathrm{c}}$ at a halo collapse redshift of $z_{\mathrm{c}} \approx 10$ approaches $\delta_{\mathrm{c}} \approx 1.62$, in good agreement with the results seen in Fig. 1. Small discrepancies are due to the fact that the power law (35) is not strictly valid unless at 


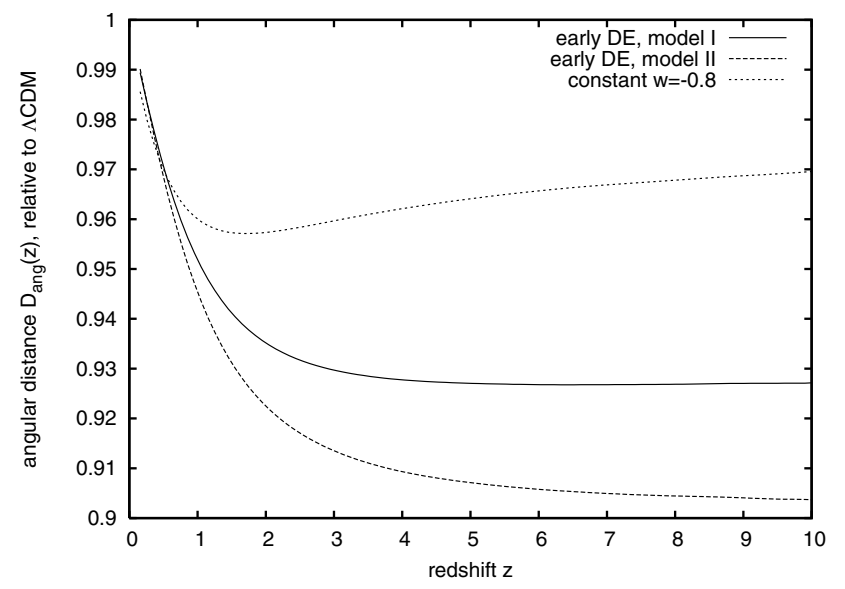

Fig. 3. The angular-diameter distance as a function of redshift is shown for two Early DE models relative to $\Lambda \mathrm{CDM}$. A model with constant $w=-0.8$ is also shown for comparison.

very high redshift, and that the initial density contrast $\Delta(x)-1$ is slightly larger in Early DE than in $\Lambda$ CDM models.

\section{Cosmological consequences}

Before we describe cosmological consequences depending on the spherical collapse parameter $\delta_{\mathrm{c}}$, we briefly summarise in Sect. 3.1 the impact of Early DE on the global geometry of the Universe. We do so to show that distance measures and the age of the Universe are only moderately affected, leaving statements depending on them virtually unchanged, such as those based on the interpretation of supernovae of type Ia. The following Sects. 3.2 to 3.5 describe measures of non-linear structure formation whose dependence on the spherical-collapse parameters and the growth factor will be detailed there.

\subsection{Distances and age}

The influence of Early DE on global geometrical properties of the Universe is illustrated in Fig. 3, which shows the angulardiameter distance $D_{\text {ang }}(z)$ in units of $c H_{0}^{-1}$ as a function of redshift relative to $\Lambda \mathrm{CDM}$. A model with constant $w=-0.8$ is shown for comparison. Note that this curve also gives the ratio of luminosity distances $D_{\text {lum }}(z)$ between DE models and $\Lambda C D M$ because $D_{\text {lum }}(z)$ and $D_{\text {ang }}(z)$ only differ by the ratio of scale factors between emission and observation.

As the figure shows, distance measures are changed only moderately, by $\lesssim 8 \%$ for source redshifts below $z=2$. The cosmic time is changed by a larger amount, as can be seen in Fig. 4. It shows the cosmic time as a function of redshift in units of $H_{0}^{-1}$, again relative to $\Lambda \mathrm{CDM}$.

The increased expansion rate of the Universe compared to $\Lambda C D M$, in particular at early times, reduces the age of the Universe by approximately $(5-10) \%$ at low redshifts. The effect of DE with constant $w=-0.8$ is substantially less pronounced.

\subsection{The mass function}

The reduced linear density contrast $\delta_{\mathrm{c}}$ necessary for spherical collapse has a pronounced influence on the mass function of DM halos. The Press-Schechter mass function for example can be

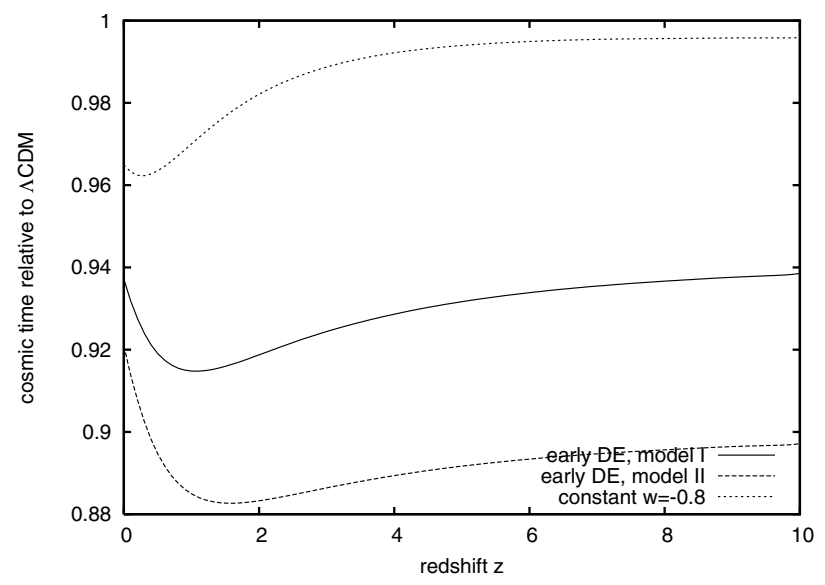

Fig. 4. Cosmic time in units of $H_{0}^{-1}$ as a function of redshift, relative to $\Lambda \mathrm{CDM}$. Three curves are given, two for the Early DE models and one for a constant $w=-0.8$ for comparison. For fixed Hubble constant $H_{0}$, Early DE makes the Universe younger today by (5-10)\% compared to $\Lambda \mathrm{CDM}$, while a fixed present age would reduce $H_{0}$.

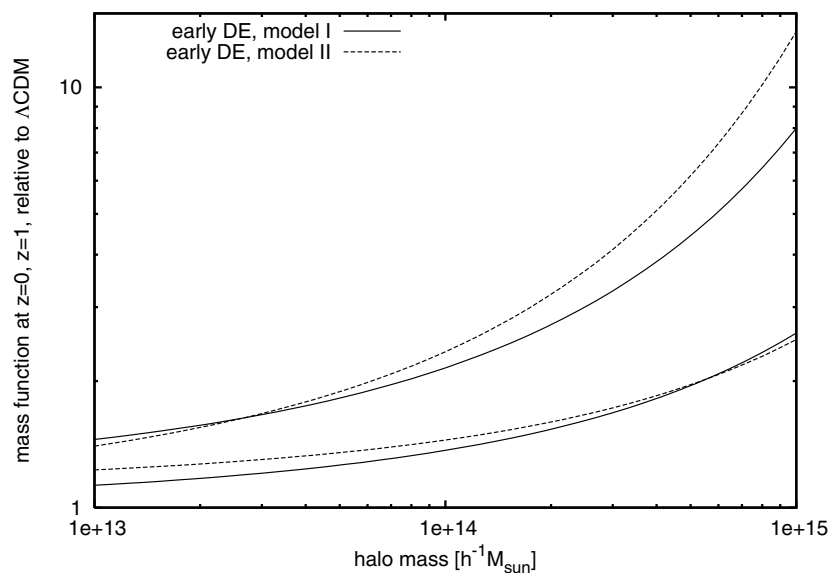

Fig. 5. Ratio between the DM halo mass function between $10^{13}$ and $10^{15} h^{-1} M_{\odot}$ in two Early DE models compared to $\Lambda$ CDM. Two pairs of curves are given, the lower for $z=0$, the upper for $z=1$. The ratio increases monotonically with increasing mass. While massive clusters today are 1.5-2.5 times more abundant in CMB-normalised Early DE compared to the $\Lambda \mathrm{CDM}$ model, their number is expected to be up to an order of magnitude higher at redshift $z=1$ in Early DE models.

written as (Press \& Schechter 1974)

$$
\begin{aligned}
N(M, z) \mathrm{d} M= & \sqrt{\frac{2}{\pi}} \frac{\rho_{0} \delta_{\mathrm{c}}(z)}{\sigma_{R} D_{+}(z)} \frac{\mathrm{d} \ln \sigma_{R}}{\mathrm{~d} M} \\
& \times \exp \left(-\frac{\delta_{\mathrm{c}}^{2}(z)}{2 \sigma_{R}^{2} D_{+}^{2}(z)}\right) \frac{\mathrm{d} M}{M}
\end{aligned}
$$

showing that $\delta_{\mathrm{c}}^{2}(z)$ is compared in the argument of the exponential function to the variance $\sigma_{R}^{2} D_{+}^{2}(z)$ of the DM density field smoothed on scales $R$ corresponding to the mass $M$, with $D_{+}(z=0)=1$. Linear quantities occur in the exponential of the Press-Schechter mass function because it predicts the distribution of the later non-linear fluctuations from their linear distribution at early times. Even small reductions of $\delta_{\mathrm{c}}$, as they are illustrated in Fig. 1, lead to a noticeable increase in the mass function for a fixed normalisation parameter $\sigma_{8}$ of the DM power spectrum. The increase of the mass function in the two Early Dark Energy models, compared to $\Lambda \mathrm{CDM}$, is illustrated in Fig. 5. 
The figure shows the mass function proposed by Sheth \& Tormen (1999), which assumes ellipsoidal rather than spherical halo collapse and reproduces the halo abundances in simulations significantly better than the original Press-Schechter mass function. As Fig. 5 shows, Early DE has a pronounced effect on the mass function. While halos with masses $\lesssim 10^{13} h^{-1} M_{\odot}$ have approximately equal abundances as in $\Lambda \mathrm{CDM}$, massive clusters with $M \gtrsim 5 \times 10^{14} h^{-1} M_{\odot}$ are of the order of 1.5-2.5 times more abundant at the present epoch, provided the models are normalised according to the CMB temperature fluctuations. At redshift unity, however, the mass functions differ by factors of $\sim(5-10)$ for massive clusters. Therefore, even if the models were normalised so as to get closer to the cluster abundance expected in $\Lambda C D M$ today, they still predicted a higher number density of high-redshift clusters. As Fig. 7 shows in more detail, the normalisation parameter would have to be lowered to $\sigma_{8} \approx 0.69$ for the Early DE models to exactly reproduce the abundance of massive halos at $z=0$ of a $\Lambda \mathrm{CDM}$ model with $\sigma=0.84$.

Although the luminosity function of hot galaxy clusters is now quite well-known (e.g. Ebeling et al. 1998; Böhringer et al. 2002; see Rosati et al. 2002 for a review), there is still considerable uncertainty in the mass function derived from it. As Pierpaoli et al. (2003) explain, cluster masses can be estimated either from luminosities or from temperatures using the appropriate scaling relations, whose scatter introduces considerable uncertainties. Taking these into account, they constrain the normalisation parameter $\sigma_{8}$ required to match the cluster abundance to $\approx 0.8$, with relative $1-\sigma$ errors of order (5-9)\%. Taking all possible systematic errors into account, Schücker et al. (2003) find error bars that are as much as two times as large. Due to the steepness of the cluster mass function, such uncertainties in $\sigma_{8}$ imply that the abundance of massive clusters is not sufficiently well-known yet to rule out the higher number densities predicted by our CMB-normalised Early DE models.

The earlier development of the mass function naturally leads to enhanced dynamical activity of DM halos, in particular at the highest cluster masses. The extended Press-Schechter formalism (Bond et al. 1991) allows the calculation of the merger probability,

$$
\frac{\mathrm{d}^{2} p}{\mathrm{~d} \ln \Delta M \mathrm{~d} t}(M, z)
$$

i.e. the probability for a halo of mass $M$ to merge within time $t$ at redshift $z$ with another halo of mass $\Delta M$ (Lacey \& Cole 1993, 1994). Multiplying expression (39) with the mass function and the differential cosmic volume $\mathrm{d} V$ corresponding to a redshift interval $\mathrm{d} z$, integrating over a mass range from $M_{1}$ to $M_{2}$, and transforming from unit time to unit redshift yields the total number of mergers per unit redshift between a halo of mass $M$ and another halo of mass between $M_{1}$ and $M_{2}$,

$\delta N(M, z)=N(M, z)\left|\frac{\mathrm{d} V}{\mathrm{~d} z}\right| \int_{\ln M_{1}}^{\ln M_{2}} \mathrm{~d} \ln M \frac{\mathrm{d}^{2} p(M, z)}{\mathrm{d} \ln M \mathrm{~d} t} \frac{\mathrm{d} t}{\mathrm{~d} z}$.

We choose three different masses $M_{2}=\left\{10^{13}, 10^{14}, 10^{15}\right\} h^{-1} M_{\odot}$ and $M_{1}=M_{2} / 4$. In other words, using expression (40) we compute the number per unit redshift of DM halos at redshift $z$ that are undergoing a major merger with another halo having at least $25 \%$ of their mass. Figure 6 shows the the merger numbers in the two Early DE models and in $\Lambda$ CDM.

The curves in the figure show that major mergers at moderate and high redshifts of DM halos are substantially more frequent in the two Early DE models compared to $\Lambda \mathrm{CDM}$. This reflects our previous result that the halo mass function grows earlier in the

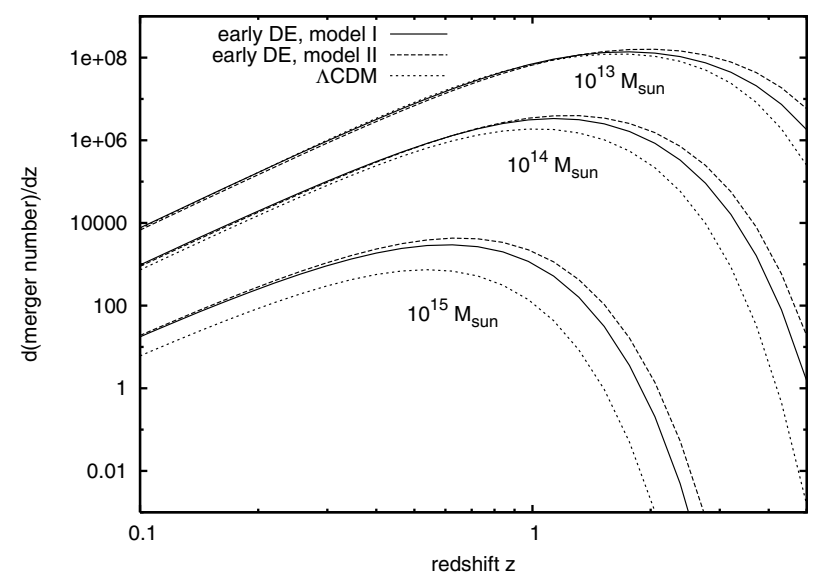

Fig. 6. The number of mergers per unit redshift at redshift $z$ is shown here for DM halos with mass $M=\left\{10^{13}, 10^{14}, 10^{15}\right\} h^{-1} M_{\odot}$ and subhalos with mass between $M / 4$ and $M$. The three curves for each halo mass show results for the two Early DE models and $\Lambda$ CDM. Reflecting the earlier growth of the mass function in Early DE models, major mergers set in earlier and are more frequent at moderate and high redshifts compared to $\Lambda \mathrm{CDM}$.

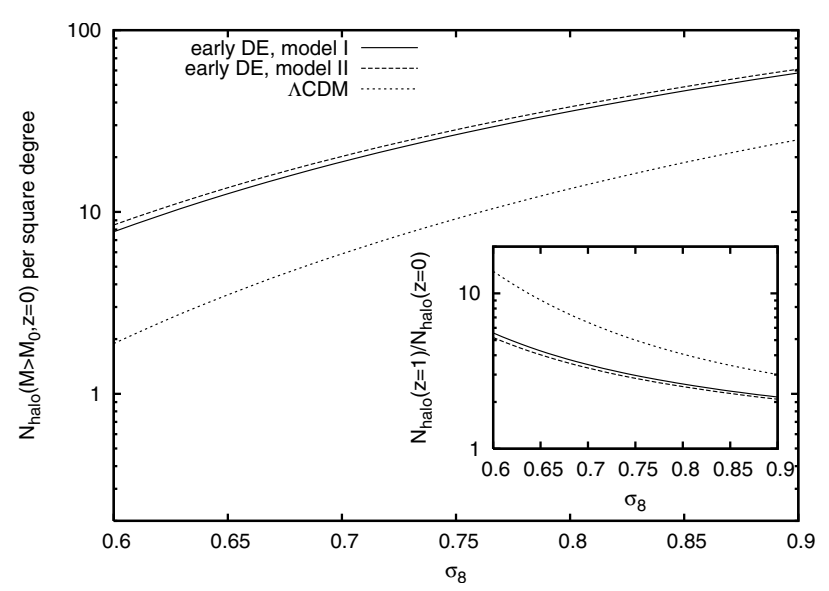

Fig. 7. Main panel: the number of halos per square degree with masses $M \geq 10^{14} h^{-1} M_{\odot}$ and redshifts $z \geq 1$ is shown as a function of the normalisation parameter $\sigma_{8}$. At $\sigma_{8} \sim 0.7$, the Early DE models have $\sim 25$ cluster-sized halos with $z \geq 0$ per square degree, for which the $\Lambda \mathrm{CDM}$ model needs $\sigma_{8} \sim 0.9$. The curves in the small panel show the ratios of halo numbers with mass $M \geq 10^{14} h^{-1} M_{\odot}$ above redshifts zero and unity for the two models of Early DE and for $\Lambda$ CDM as a function of the normalisation parameter $\sigma_{8}$. At fixed $\sigma_{8} \sim 0.8$, the number of cluster-sized halos grows by approximately twice as much in the $\Lambda \mathrm{CDM}$ compared to the Early DE models.

Early DE models, leading to enhanced merger activity at earlier times.

Benson et al. (2005) argued that the approach of the extended Press-Schechter theory is flawed in the sense that the predicted merger rate is asymmetric in the masses of the merging halos and proposed an alternative approach based on the coagulation equation. However, the asymmetry of the extended Press-Schechter approach is only important for mass ratios of the merging halos much different from unity and should thus be unimportant in our context.

Of course, these results on the abundance of DM halos and the frequency of major mergers depend on and are very sensitive to the normalisation of the power spectrum, as the curves in the main panel of Fig. 7 illustrate. Much less dependent on the amplitude of the power spectrum is the expected ratio of halo 
numbers above two different redshifts. The curves in the small panel of Fig. 7 give examples.

The curves show, for three cosmological models by how much the number of cluster-sized halos with mass $M \geq$ $10^{14} h^{-1} M_{\odot}$ grows between redshifts unity and zero dependent on the normalisation parameter $\sigma_{8}$. For $\sigma_{8}=0.8$ in $\Lambda \mathrm{CDM}$, there are about four times more clusters today than there were at redshift unity and about two times more in the Early DE models. This corroborates that clusters are more abundant at high redshifts in the Early DE models compared to $\Lambda$ CDM. Since CDM is a hierarchical model of structure formation, a similar effect appears for lower-mass halos at higher redshift. In the Early DE models, for instance, the number of galaxy-sized halos evolves much less than in $\Lambda C D M$ above redshifts 2 and beyond.

\subsection{Halo properties}

Earlier work has found that DM halos tend to be more concentrated if they form earlier, where the concentration $c$ is defined to be the ratio between the halo's virial and scale radii (Navarro et al. 1997; Bullock et al. 2001; Eke et al. 2001). While the central slope of DM density profiles in halos is still under some debate (cf. Power et al. 2003), numerical simulations consistently show that halos in (Cold) DM have a steep density profile outside and a flat density profile inside the scale radius. The definition of the virial radius changes in the literature, depending on whether the mean density enclosed by the virial radius is supposed to be 200 or another factor times the mean or the critical cosmic density. Different recipes have also been given for the statistical relation between the virial mass and the concentration of halos. They were tested against numerical results on DE models by Dolag et al. (2004) who found that the algorithm described by Eke et al. (2001) worked for a variety of DE models without adaptation.

Models tested by Dolag et al. (2004) included one with constant equation-of-state parameter $w=-0.6$ and two with variable $w$ derived from the Ratra-Peebles (Ratra \& Peebles 1988) and SUGRA (Brax \& Martin 2000) potentials. The model using the Ratra-Peebles potential results in a slow evolution of $w$ with redshift, while the model using the SUGRA potential results in a rapid one. Since the simulations thus probed a broad range of equations-of-state and found very good agreement of the halo concentrations with the prediction according to the algorithm by Eke et al. (2001), we can be confident that this algorithm may also be used for estimating halo concentrations in Early DE models.

Two things that all algorithms have in common is that they have to define a collapse redshift $z_{\mathrm{c}}$ for a halo of given mass $M$ at redshift $z$ and a ratio between the central density of the halo and the mean or critical cosmic background density at $z_{\mathrm{c}}$. Eke et al. (2001) define the collapse redshift by requiring that, at $z_{\mathrm{c}}$, the amplitude of the power spectrum at the mass scale of the halo reaches a given fixed value. Thus, it is the growth factor $D_{+}(z)$ that renders the so-defined halo concentrations sensitive to Early DE. We show in Fig. 8 the expected concentration according to Eke et al.'s prescription as a function of halo mass at redshifts zero (upper curves) and unity (lower curves).

In agreement with earlier studies (Bartelmann et al. 2002; Klypin et al. 2003; Dolag et al. 2004; Weinberg \& Kamionkowski 2003), halos in cosmologies with dynamical DE tend to be more concentrated than in $\Lambda \mathrm{CDM}$, reflecting their earlier growth on a cosmological background with higher mean density. For galaxy-sized halos in the Early DE models, the concentration increases by $\approx 20 \%$ at redshift zero.

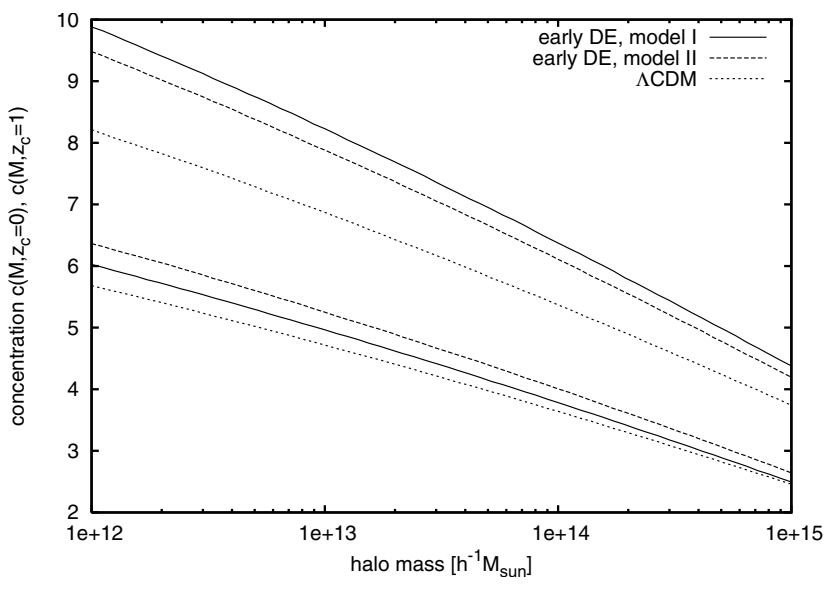

Fig. 8. Halo concentrations as functions of mass, according to Eke et al.'s algorithm, for redshifts at unity (lower curves) and zero (upper curves). Results for the two Early DE models and for $\Lambda$ CDM are given. Halos in Early DE models are more concentrated than in $\Lambda \mathrm{CDM}$, and the difference increases for smaller masses.

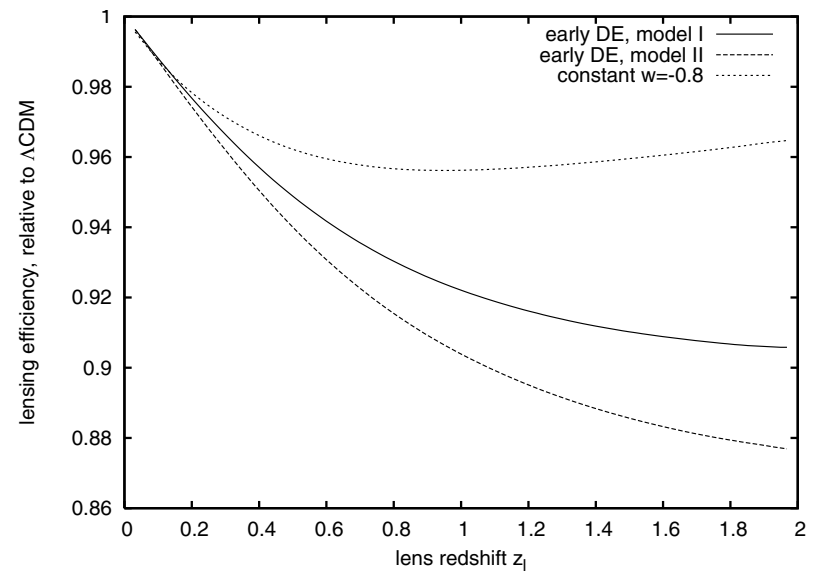

Fig. 9. The effective lensing distance is plotted here for the two Early DE models, normalised by the result in $\Lambda$ CDM. The curve for constant $w=-0.8$ is shown for comparison. Sources are assumed at redshift $z_{\mathrm{s}}=2$. The effective lensing distance is lower by $\approx 5 \%$ in Early DE than in $\Lambda$ CDM models at typical lens redshifts near $z=0.4$ and by $\lesssim 10 \%$ at redshifts near unity.

\subsection{Gravitational lensing}

The efficiency of gravitational lensing by isolated lenses such as galaxies or galaxy clusters is given by the critical surface-mass density

$\Sigma_{\mathrm{cr}}=\frac{c^{2}}{4 \pi G} \frac{D_{\mathrm{s}}}{D_{\mathrm{l}} D_{\mathrm{ls}}}$

which contains the angular-diameter distances $D_{\mathrm{l}, \mathrm{s}, \mathrm{ls}}$ from the observer to the lens and to the source, and from the lens to the source, respectively (e.g. Schneider et al. 1992). The effective lensing distance $D_{\text {eff }} \equiv D_{1} D_{\mathrm{ls}} / D_{\mathrm{s}}$ thus measures the geometrical efficiency of a given mass distribution.

$\mathrm{DE}$ reduces the effective lensing distance compared to $\Lambda \mathrm{CDM}$. For sources at redshift $z_{\mathrm{s}}=2$, as assumed for the figure, identical mass distributions are $\approx 5 \%$ less efficient at $z_{1} \approx 0.4$, and $\approx 10 \%$ less efficient at $z_{1} \approx 1.0$, in Early DE than in $\Lambda$ CDM models. Compared to the increased concentration of the DM halos, this effect is less important for strong lensing by galaxies, groups, or clusters (Meneghetti et al. 2005). 


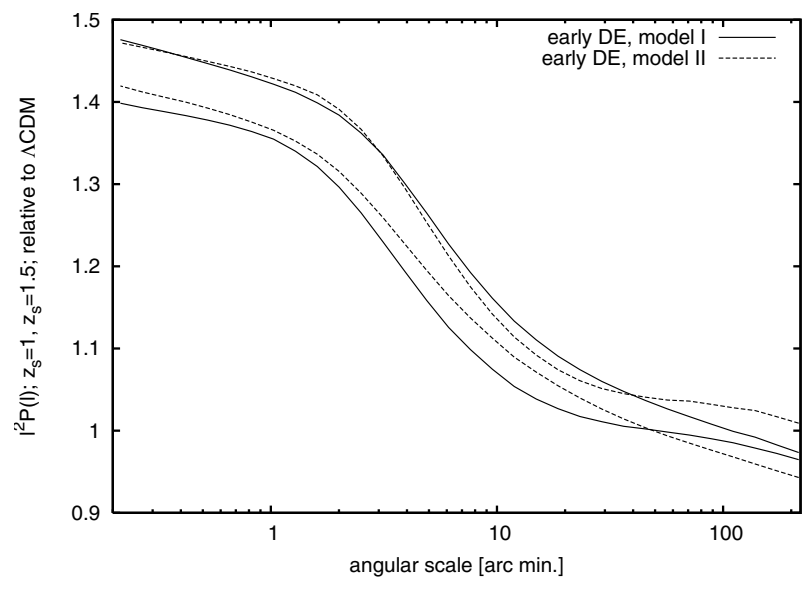

Fig. 10. The weak-lensing power, expressed by means of the convergence power spectrum as $l^{2} P_{K}(l)$, is plotted as a function of angular scale for the two Early DE models for the two different source redshifts $z_{\mathrm{s}}=1$ and 1.5. The curves are normalised by the expectation in $\Lambda \mathrm{CDM}$.

In sufficient approximation, weak gravitational lensing by large-scale structures can be described by the power spectrum $P_{K}(l)$ of the effective convergence $\kappa$ (see Bartelmann \& Schneider 2001 for a review). The power spectrum for $\kappa$ is a line-of-sight integral over the DM power spectrum, weighted by a combination of distance factors similar to those appearing in the critical surface-mass density Eq. (41). Therefore, weak lensing is affected by Early DE mainly through the growth factor $D_{+}(z)$, and to a lesser degree by the modified angular-diameter distances. Figure 10 shows how Early DE changes $P_{K}(l)$.

The weak-lensing power is illustrated there plotting $l^{2} P_{K}(l)$ as a function of angular scale for the two Early DE models, relative to the $\Lambda$ CDM model. Two pairs of curves are given for the two source redshifts $z_{\mathrm{s}}=1$ and 1.5 . The curves tend to unity at angular scales above 10 arc minutes, indicating that weak lensing will not be modified compared to $\Lambda \mathrm{CDM}$ on large angular scales. On small angular scales $\lesssim 1^{\prime}$, however, the weak-lensing power in the Early DE models exceeds that in the $\Lambda$ CDM model by $\sim 40 \%$ for $z_{\mathrm{s}}=1$ and by $\sim 55 \%$ for $z_{\mathrm{s}}=1.5$. This would imply that $\sigma_{8}$, as derived from weak-lensing measurements, could be $\sim 1.4^{-1 / 2} \approx 0.8$ times smaller than inferred assuming a $\Lambda \mathrm{CDM}$ model.

\subsection{Number counts of thermal Sunyaev-Zel'dovich clusters}

The most significant observable consequence of Early DE models identified so far is that the counts of cluster-sized halos should decrease significantly more slowly than in $\Lambda \mathrm{CDM}$ (cf. Figs. 5 and 7). While statistically complete galaxy-cluster samples are notoriously hard to identify, future cluster surveys based on the thermal Sunyaev-Zel'dovich (SZ) effect (Sunyaev $\&$ Zeldovich 1972) should provide ideally suitable data for testing this prediction of Early DE models. Like the number counts of dark-matter halos, those of thermal-SZ clusters are changed by Early $\mathrm{DE}$, mainly through the linear overdensity $\delta_{\mathrm{c}}$, and to some degree also by the modified cosmic geometry.

The upcoming Planck satellite, due for launch in 2007, will observe the entire microwave sky in frequency bands between 30 and $857 \mathrm{GHz}$ with an angular resolution of as few as 5 arc minutes. One of its frequency bands is centred on $217 \mathrm{GHz}$ where the (non-relativistic) thermal SZ effect vanishes. Planck's frequency bands are thus well-placed for identifying the unique spectral signature of the thermal SZ effect that reduces the CMB

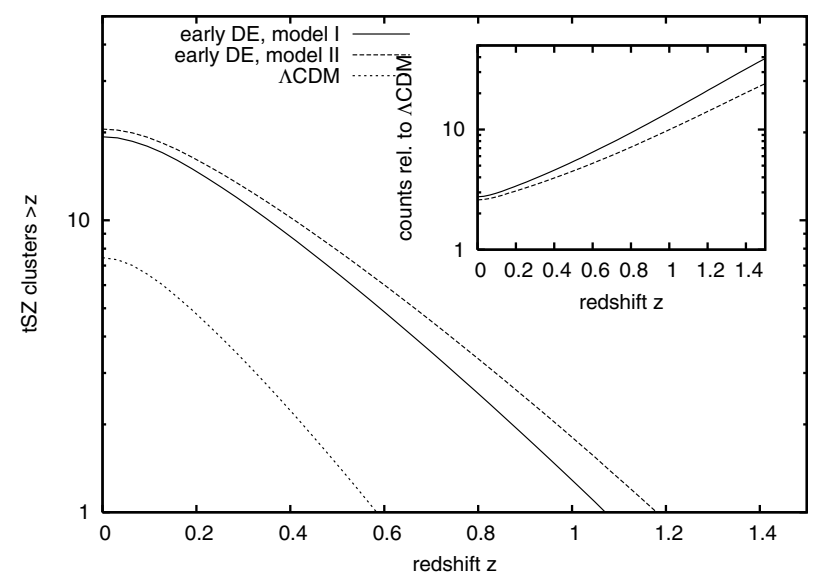

Fig. 11. Main panel: cumulative redshift distribution of clusters expected to be detected through their thermal SZ effect by the Planck satellite. Curves are shown for the two models of Early DE and for $\Lambda C D M$, and they are normalised to show the number of clusters per 10 square degrees. Thermal-SZ cluster counts in the Early DE models start at $z=0$ at approximately twice the value in $\Lambda \mathrm{CDM}$ and reach to substantially higher redshifts. The curves in the small panel show the redshift distributions in the Early DE models, divided by the expectation in the $\triangle C D M$ model. For $z \geq 0$, the total number of detections should increase by a factor of $2-3$, while the number of clusters at redshifts $\geq 1$ should be about an order of magnitude higher in Early DE compared to $\Lambda \mathrm{CDM}$ models.

intensity below, and increases it above, $217 \mathrm{GHz}$. Planck's high sensitivity and comparatively high angular resolution will enable it to detect a huge sample of clusters reaching high redshift.

Analytic estimates raise the expectation that Planck may find a number of the order of 30000 galaxy clusters on the full sky (da Silva et al. 2000; Bartelmann et al. 2003), while simulations taking realistic foreground contamination, noise patterns, and full-sky, multi-band filtering techniques into account arrive at lower numbers, $\lesssim 10000$ (Schäfer et al. 2004). Nonetheless, the cluster sample expected from Planck will be enormous. Following the analytic description of the Planck cluster sample given in Bartelmann et al. (2003), we show in Fig. 11 the cumulative redshift distribution of clusters in the sample expected from Planck in the Early DE models, normalised by the expectation in the $\Lambda$ CDM model.

While the total number of detections is moderately increased by a factor of two to three, the number of high-redshift clusters detectable in Early DE models is substantially higher than in $\Lambda \mathrm{CDM}$. The number of clusters above redshift unity is already an order of magnitude larger. Thus, the number of clusters detected by Planck will provide a highly sensitive test for Early DE compared to $\Lambda$ CDM models.

Interestingly, observations of the $\mathrm{CMB}$ on small angular scales $\left(\lesssim 5^{\prime}\right)$ with the Cosmic Background Imager (CBI) indicate a much higher temperature fluctuation amplitude than expected in $\Lambda \mathrm{CDM}$ with the normalisation $\sigma_{8} \sim 0.8$ typically inferred from other observations (Padin et al. 2001; Mason et al. 2003). If due to the thermal SZ effect, these measurements require $\sigma_{8} \gtrsim 1$ in the $\Lambda \mathrm{CDM}$ model (Bond et al. 2005), which would stretch other normalisation constraints to their limits. Cosmological models with Early DE such as those used here as examples could naturally explain this "CBI anomaly": our Early DE models reach the same abundance of thermal SZ clusters with $\sigma_{8} \approx 0.8$ for which the $\Lambda$ CDM model needs $\sigma_{8} \approx 1.0$, which is necessary for removing the $\mathrm{CBI}$ anomaly according to Bond et al. (2005). 


\section{Summary and conclusions}

We have investigated expectations for the growth of non-linear structures in a model universe with Early DE. Such models assume that the accelerated cosmic expansion is driven by a scalar field that has non-negligible energy density all through cosmic history. We have selected two examples for such models with Early Dark Energy whose parameters are chosen so as to comply with the temperature fluctuations measured in the cosmic microwave background.

We have generalised the spherical-collapse model such that $\mathrm{DE}$ with the variable equation-of-state parameter $w$ can be taken into account. In our models, the DE follows for $z \gtrsim 1$ the density of the dominant component in the mixture of cosmic fluids. Modified in this way, the spherical collapse model predicts that the linear density contrast necessary for collapse, $\delta_{\mathrm{c}}$, is lowered compared to $\Lambda$ CDM models or DE models with constant $w$.

This first result, which may appear unexpected, can be understood as follows. The requirement of having virialised halos at a given redshift sets the initial overdensity inside the spherical perturbation, idealising the later halo. Early DE reduces the linear growth factor compared to $\Lambda \mathrm{CDM}$ due to its higher expansion rate in the young universe. The initial overdensity required for later collapse is thus extrapolated to lower linear overdensity at collapse time. Lower linear density contrast is thus sufficient for non-linear collapse, which increases the number of fluctuations capable of forming halos.

The effects of Early DE on the geometrical properties of the Universe are moderate. Assuming the same Hubble constant today, such models are $\sim 5 \%$ younger than comparable $\Lambda \mathrm{CDM}$ models are today, and the angular-diameter distances are lowered by no more than $\sim 10 \%$, even to high redshifts. Yet, these effects are noticeably stronger than in DE models with constant $w$; typically, they are roughly twice as large as in a model universe that has $w=-0.8$.

Since halos tend to grow earlier in DE compared to $\Lambda \mathrm{CDM}$ models, their core densities are higher, and so are their concentration parameters. Using the prescription of Eke et al. for computing expected halo concentrations, we found that halos at redshift zero are more concentrated than in $\Lambda$ CDM by $\sim 20 \%$ for galaxy masses and by $\sim 15 \%$ for cluster masses. This increase is visible, but smaller, for halos forming at higher redshift.

Strong gravitational lensing profits non-linearly from higher halo concentrations. On the other hand, the effective lensing distance (proportional to the inverse critical surface density for lensing) is lowered by Early DE, albeit weakly. At redshifts that are typical for strong-lensing galaxies or clusters, $\sim 0.3-0.8$, say, we found a reduction of the effective lensing distances of order $\sim 8 \%$ compared to $\Lambda$ CDM. The power of weak gravitational lensing by large-scale structures, however, is more substantially changed. While there is no difference in the weaklensing power between Early DE and $\Lambda$ CDM models on large angular scales $\gtrsim 1^{\circ}$, it is increased by $\sim 40 \%$ on arc-minute scales for sources at redshift $\sim 1$. This implies that e.g. the two-point correlation function of the cosmic shear would increase more steeply with decreasing angular scale than expected in $\Lambda$ CDM. It also implies that the normalisation of the DM power spectrum, $\sigma_{8}$, inferred from weak lensing, should be lowered by a factor of $\sim 0.8$ as compared to its value for a $\Lambda$ CDM model.

The most pronounced effect, however, is in regard to the present number density of massive halos and its evolution towards higher redshift. The Early DE models we have studied here, normalised to the CMB temperature-fluctuation measurements, predict approximately the same number density of galaxy-sized halos today that is expected in a $\Lambda \mathrm{CDM}$ universe and a number density of cluster-sized halos that is $\sim 40 \%$ higher. Given the uncertainties in cluster counts even at low redshift, this appears tolerable, although a very moderate reduction of $\sigma_{8}$ would establish complete agreement at redshift zero between the halo counts in Early DE and $\Lambda$ CDM models. However, this present cluster population shrinks much less quickly towards high redshift than in $\Lambda \mathrm{CDM}$, implying that many more of the clusters existing today were already present at redshifts of the order of unity.

The cluster population above a mass limit of $10^{14} h^{-1} M_{\odot}$ expected for $\sigma_{8}=0.8$ in Early DE models shrinks by a factor of $\sim 2.5$ at redshift unity compared to redshift zero, but by a factor of $\sim 4$ in $\Lambda$ CDM models. This discrepancy increases rapidly for more massive clusters. Raising the mass limit to $5 \times 10^{14} h^{-1} M_{\odot}$ makes the cluster population shrink by a factor of $\sim 9$ in the Early DE models, but by a factor of $\sim 28$ in an equally normalised $\Lambda$ CDM model.

It appears reasonable here to distinguish two possibly different values of $\sigma_{8}$ extracted from linear and non-linear structure growth. As the mass function (38) shows, lowering $\delta_{\mathrm{c}}$ due to Early DE can be compensated by equally lowering $\sigma_{8}$. This implies that approximately the same halo number at all redshifts as in the $\Lambda \mathrm{CDM}$ model can be reproduced in the Early DE models by suitably lowering $\sigma_{8}$. This $\sigma_{8}$ would then properly describe the abundance of non-linear, collapsed structures. This can be tested against the amplitude of linear structures, which is measurable for instance by the galaxy power spectrum on large scales or weak gravitational lensing on scales $\gtrsim 10^{\prime}$. In other words, if the value of $\sigma_{8}$ extracted from non-linear structures within a $\Lambda \mathrm{CDM}$ model is systematically higher than the $\sigma_{8}$ inferred from larger structures, this would clearly hint at the presence of Early DE.

For a given $\sigma_{8}$ measured from linear fluctuations, the Early DE models predict a substantially slower evolution of the halo population than in the $\Lambda \mathrm{CDM}$ model. This prediction has several immediate observable consequences. First, the evolution of the $\mathrm{X}$-ray cluster luminosity function towards redshift unity should be somewhat flatter than expected in a $\Lambda \mathrm{CDM}$ universe. Current data are probably still insufficient for quantitative tests. Second, the dynamical activity of clusters near redshift unity should be substantially higher than expected in $\Lambda \mathrm{CDM}$. The number of major mergers experienced by cluster-sized halos near redshift unity is predicted to be $\sim 5$ times higher than in $\Lambda \mathrm{CDM}$. Third, and most prominently, the number of clusters expected to be found in thermal SZ surveys should increase substantially. Applying a simple model for cluster detection by the Planck satellite, we found that $\sim 2$ times more clusters should be found at arbitrary redshifts by Planck than in a $\Lambda$ CDM universe, but $\sim 10$ times more above redshift unity. Leaving the question aside of how redshifts could be measured for clusters detected exclusively by their thermal SZ effect, this prediction will be testable very soon. We note that this consequence of Early DE may naturally explain the high fluctuation amplitude of CMB temperature fluctuations on arc-minute scales, the so-called "CBI anomaly" (Padin et al. 2001; Mason et al. 2003; Bond et al. 2005).

Given these results, we arrive at the main conclusion that models for Early DE will leave a measurable imprint on weak-lensing measurements and determinations of the distanceredshift relation that is measured using type-Ia supernovae, but their most distinguishing signature is the slow evolution of the cluster abundance between redshifts $\sim 1$ and zero. Structure formation being hierarchical, this also implies a similar effect on 


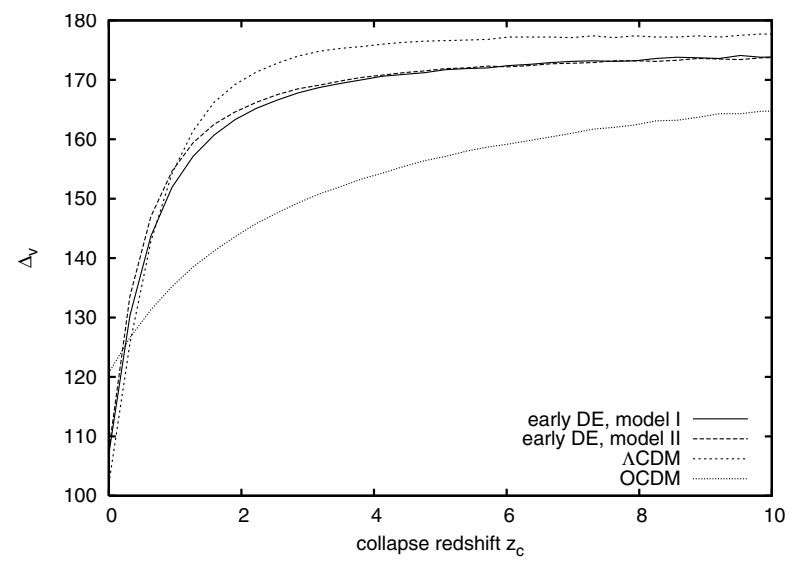

Fig. A.1. The virial overdensity $\Delta_{v}$ is shown as a function of collapse redshift $z_{\mathrm{c}}$ for the same four cosmologies used in Fig. 1. The change in $\Delta_{\mathrm{V}}$ due to Early DE is small compared to the difference between the more conventional models.

the abundance evolution of galaxy-sized halos at substantially higher redshifts, $z \gtrsim 2-3$.

Acknowledgements. We are grateful to E. Thommes for helpful discussions.

\section{Appendix A: The virial overdensity}

The conventional calculation used in many papers (see e.g. Lahav et al. 1991; Kitayama \& Suto 1996; Wang \& Steinhardt 1998; Weinberg \& Kamionkowski 2003; Horellou \& Berge 2005; Maor \& Lahav 2005; Mota \& van de Bruck 2004) starts from the Friedmann equation (4) for the second time derivative $\ddot{y}$ of the perturbation radius and converts it to an energy equation after multiplication with $\dot{y}$. This energy equation contains the cosmological constant or DE density in a term that appears analogous to a potential-energy term. Energy conservation is then used by equating the total potential energy of the over-dense sphere at turn-around to the sum of kinetic and potential energies at virialisation, which can be expressed solely in terms of the potential energy by means of the virial theorem.

Following this approach, assuming that the DE does not clump and therefore not participate in the virialisation of the collapsing overdensity either (see Maor \& Lahav (2005) for a thorough discussion), we calculate $\Delta_{\mathrm{V}}$ and confirm that it is only weakly modified by Early DE compared to, e.g. the $\Lambda \mathrm{CDM}$ result.

We show in Fig. A.1 the virial overdensity $\Delta_{\mathrm{V}}$ calculated in this way as a function of the collapse redshift $z_{\mathrm{c}}$ for two models of Early DE and compare it to the results for a $\Lambda \mathrm{CDM}$, with $\Omega_{\mathrm{m}, 0}=0.3$ and $\Omega_{\Lambda, 0}=0.7$, and an OCDM model, with $\Omega_{\mathrm{m}, 0}=$ 0.3 . While $\Delta_{\mathrm{V}}$ is somewhat lower at moderate and high $z_{\mathrm{c}}$ for the Early DE models than for $\Lambda \mathrm{CDM}$, the difference between them remains moderate. For high $z_{\mathrm{c}}$, they approach the "canonical" value of $18 \pi^{2} \approx 178$ and fall towards $\Delta_{\mathrm{v}} \approx 110$ for $z_{\mathrm{c}}=0$. As shown for comparison, $\Delta_{\mathrm{V}}$ shows a substantially flatter behaviour with $z_{\mathrm{c}}$ in the OCDM model.

We have, however, serious doubts about whether this approach is valid in the context of cosmological models with (Early) DE. The virial theorem concerns time-averaged energies of particles in bound orbits. Dark-matter particles orbiting within collapsing overdensities, however, will not feel any force from the DE because the latter is homogeneously distributed. Thus, it appears dubious to assign a potential to the DE whose gradient should appear as a conservative force term in the equation of motion. Rather, the DE should only appear to act on the expansion of the background, and therefore it should function as a dissipative force term that should time-average out of the virial theorem. In this picture, the concept of energy conservation also needs to be revisited. Since none of our later results depends on $\Delta_{\mathrm{V}}$, we leave the discussion at that point, showing $\Delta_{\mathrm{V}}$ as the conventional approach predicts it for the Early DE models, but expressing qualms about the validity of the underlying physical concepts.

\section{References}

Bartelmann, M., \& Schneider, P. 2001, Phys. Rep., 340, 291

Bartelmann, M., Perrotta, F., \& Baccigalupi, C. 2002, A\&A, 396, 21

Bartelmann, M., Meneghetti, M., Perrotta, F., Baccigalupi, C., \& Moscardini, L. 2003, A\&A, 409, 449

Benson, A. J., Kamionkowski, M., \& Hassani, S. H. 2005, MNRAS, 357, 847 Böhringer, H., Collins, C., Guzzo, L., et al. 2002, ApJ, 566, 93 Bond, J. R., Cole, S., Efstathiou, G., \& Kaiser, N. 1991, ApJ, 379, 440 Bond, J. R., Contaldi, C. R., Pen, U.-L., et al. 2005, ApJ, 626, 12 Brax, P., \& Martin, J. 2000, PRD, 61, 103502

Bullock, J. S., Kolatt, T. S., Sigad, Y., et al. 2001, MNRAS, 321, 559

Caldwell, R. R. 2002, Physics Letters B, 545, 23

Caldwell, R. R., Dave, R., \& Steinhardt, P. J. 1998, Phys. Rev. Lett., 80, 1582

Caldwell, R. R., Doran, M., Müller, C. M., Schäfer, G., \& Wetterich, C. 2003, ApJ, 591, L75

da Silva, A. C., Barbosa, D., Liddle, A. R., \& Thomas, P. A. 2000, MNRAS, 317,37

Dolag, K., Bartelmann, M., Perrotta, F., Baccigalupi, C., et al. 2004, A\&A, 416, 853

Doran, M., \& Jäckel, J. 2002, PRD, 66, 043519

Doran, M., Lilley, M., Schwindt, J., \& Wetterich, C. 2001a, ApJ, 559, 501 Doran, M., Schwindt, J., \& Wetterich, C. 2001b, Phys. Rev. D, 64, 123520

Ebeling, H., Edge, A. C., Bohringer, H., et al. 1998, MNRAS, 301, 881

Eke, V. R., Navarro, J. F., \& Steinmetz, M. 2001, ApJ, 554, 114

Ferreira, P. G., \& Joyce, M. 1998, PRD, 58, 023503

Goldstein, J. H., Ade, P. A. R., Bock, J. J., et al. 2003, ApJ, 599, 773

Hawkins, E., Maddox, S., Cole, S., Lahav, O., et al. 2003, MNRAS, 346, 78

Horellou, C., \& Berge, J. 2005, MNRAS, 360, 1393

Kitayama, T., \& Suto, Y. 1996, ApJ, 469, 480

Klypin, A., Macciò, A. V., Mainini, R., \& Bonometto, S. 2003, ApJ, 599, 31

Lacey, C., \& Cole, S. 1993, MNRAS, 262, 627

Lacey, C., \& Cole, S. 1994, MNRAS, 271, 676

Lahav, O., Lilje, P. B., Primack, J. R., \& Rees, M. J. 1991, MNRAS, 251, 128

Liddle, A. R., \& Scherrer, R. J. 1999, PRD, 59, 023509

Linder, E. V., \& Jenkins, A. 2003, MNRAS, 346, 573

Ma, C., Caldwell, R. R., Bode, P., \& Wang, L. 1999, ApJ, 521, L1

Maor, I., \& Lahav, O. 2005, JCAP, 0507, 003

Mason, B. S., Pearson, T. J., Readhead, A. C. S., et al. 2003, ApJ, 591, 540

Meneghetti, M., Bartelmann, M., Dolag, K., et al. 2005, A\&A, 442, 413

Mota, D. F., \& van de Bruck, C. 2004, A\&A, 421, 71

Navarro, J. F., Frenk, C. S., \& White, S. D. M. 1997, ApJ, 490, 493

Padin, S., Cartwright, J. K., Mason, B. S., et al. 2001, ApJ, 549, L1

Pierpaoli, E., Borgani, S., Scott, D., et al. 2003, MNRAS, 342, 163

Power, C., Navarro, J. F., Jenkins, A., et al. 2003, MNRAS, 338, 14

Press, W. H., \& Schechter, P. 1974, ApJ, 187, 425

Ratra, B., \& Peebles, P. J. E. 1988, PRD, 37, 3406

Readhead, A. C. S., Mason, B. S., Contaldi, C. R., et al. 2004, ApJ, 609, 498

Rebolo, R., Battye, R. A., Carreira, P., et al. 2004, MNRAS, 353, 747

Riess, A. G., Strolger, L.-G., Tonry, J., Casertano, S., et al. 2004, ApJ, 607, 665

Rosati, P., Borgani, S., \& Norman, C. 2002, ARA\&A, 40, 539

Schäfer, B. M., Pfrommer, C., Hell, R., \& Bartelmann, M. 2004, [arXiv:astro-ph/0407090]

Schneider, P., Ehlers, J., \& Falco, E. E. 1992, Gravitational Lenses (Heidelberg: Springer Verlag)

Schücker, P., et al. 2003, A\&A, 398, 867

Sheth, R. K., \& Tormen, G. 1999, MNRAS, 308, 119

Spergel, D. N., Verde, L., Peiris, H. V., Komatsu, E., et al. 2003, ApJS, 148, 175 Sunyaev, R. A., \& Zeldovich, Y. B. 1972, Comm. Astrophys. Space Phys., 4, 173 Tegmark, M., Strauss, M., Blanton, M., Abazajian, S., et al. 2004, PRD, 69, 103501

Wang, L., \& Steinhardt, P. J. 1998, ApJ, 508, 483

Weinberg, N. N., \& Kamionkowski, M. 2003, MNRAS, 341, 251

Wetterich, C. 1988, Nucl. Phys. B, 302, 668

Wetterich, C. 2004, Phys. Lett. B, 594, 17

Zlatev, I., \& Steinhardt, P. J. 1999, Phys. Lett. B, 459, 570 